\title{
Successive Zero-forcing DPC with Sum Power Constraint: Low-complexity Optimal Designs
}

\author{
Le-Nam Tran*, Markku Juntti*, Mats Bengtsson ${ }^{\dagger}$, and Björn Ottersten ${ }^{\dagger \ddagger}$ \\ ${ }^{*}$ Centre for Wireless Communications and Dept. Commun. Eng., University of Oulu, Oulu, Finland \\ Email: \{le.nam.tran;markku.juntti\}@ee.oulu.fi \\ ${ }^{\dagger}$ ACCESS Linnaeus Center, Royal Institute of Technology (KTH), Stockholm, Sweden \\ Email: \{mats.bengtsson,bjorn.ottersten\}@ee.kth.se \\ $\ddagger$ Interdisciplinary Centre for Security, Reliability and Trust, University of Luxembourg, Luxembourg
}

\begin{abstract}
Successive zero-forcing dirty paper coding (SZFDPC) is a simplified alternative to DPC for MIMO broadcast channels (MIMO BCs). In the SZF-DPC scheme, the noncausallyknown interference is canceled by DPC, while the residual interference is suppressed by the $\mathrm{ZF}$ technique. Due to the $\mathrm{ZF}$ constraints, the precoders are constrained to lie in the null space of a matrix. For the sum rate maximization problem under a sum power constraint, the existing precoder designs naturally rely on the singular value decomposition (SVD). The SVD-based design is optimal but needs high computational complexity. Herein, we propose two low-complexity optimal precoder designs for SZFDPC, all based on the QR decomposition (QRD), which requires lower complexity than SVD. The first design method is an iterative algorithm to find an orthonormal basis of the null space of a matrix that has a recursive structure. The second proposed method, which will be shown to require the lowest complexity, results from applying a single $Q R D$ to the matrix comprising all users' channel matrices. We analytically and numerically show that the two proposed precoder designs are optimal.
\end{abstract}

\section{INTRODUCTION}

In multiple-input multiple-output broadcast channels (MIMO BCs), a multiple-antenna base station (BS) is able to serve several multiple-antenna receivers simultaneously, thanks to the spatial multiplexing capacity. Theoretical studies have shown that the capacity region of a MIMO BC is achieved by dirty paper coding (DPC) [1]. For the sum capacity maximization problem under a sum power constraint, several iterative algorithms have been proposed to find the optimal input covariance matrices, based on the duality between the MIMO multiple access channel (MAC) and the MIMO BC [2], [3]. Generally, these algorithms suffer from high computational complexity. Thus, it is of particular interest to develop suboptimal solutions to DPC.

A suboptimal alternative to DPC for MIMO BCs, known as SZF-DPC, was introduced in [4]. In the SZF-DPC scheme, for the $k$ th user, the interference caused by users 1 to $k-1$ is canceled by DPC, and that caused by users $k+1$ to $K$ is eliminated by the $\mathrm{ZF}$ technique, where $K$ is the number of users. Let $\boldsymbol{W}_{k}$ be the precoder of the $k$ th user. Then, the

This research has been supported by Tekes, the Finnish Funding Agency for Technology and Innovation, Nokia Siemens Networks, Renesas Mobile Europe, Elektrobit, Xilinx, Academy of Finland, and partly by the European Research Council under the European Community's Seventh Framework Programme (FP7/2007-2013) / ERC grant agreement no. 228044.
ZF constraints impose that $\boldsymbol{H}_{j} \boldsymbol{W}_{k}=\mathbf{0}$ for all $j<k$, where $\boldsymbol{H}_{j}$ is the channel matrix between the BS and the $j$ th user. Naturally, we can write $\boldsymbol{W}_{k}=\boldsymbol{B}_{k} \boldsymbol{D}_{k}$, where $\boldsymbol{B}_{k}$ is designed to satisfy the ZF constraints, and $\boldsymbol{D}_{k}$ is optimized under the power constraints. The existing precoder design methods mainly differ in the way to find $\boldsymbol{B}_{k}$.

For single-antenna receivers, SZF-DPC reduces to ZF-DPC, for which the QRD-based design was proposed in [5]. The optimality of the QRD-based design for ZF-DPC is established in [6]. Applying this QRD-based approach to the precoder design for SZF-DPC may result in a suboptimal design, since the QRD-based approach essentially treats each antenna of a particular user as if it were actually a separate user. For multipleantenna receivers, in [4], a design method was proposed based on the SVD. Specifically, $\boldsymbol{B}_{k}$ is chosen as an orthonormal basis of $\mathcal{N}\left(\overline{\boldsymbol{H}}_{k}\right)$, where $\overline{\boldsymbol{H}}_{k}=\left[\boldsymbol{H}_{1}^{H} \boldsymbol{H}_{2}^{H} \cdots \boldsymbol{H}_{k-1}^{H}\right]^{H}$, and $\mathcal{N}(\boldsymbol{H})$ denotes the null space of $\boldsymbol{H}$. To find $\boldsymbol{B}_{k}$, the method in [4] applies an SVD to $\overline{\boldsymbol{H}}_{k}$. Herein, we propose two lowcomplexity precoder designs for SZF-DPC, using the QRD. First we note that it is computationally cheaper to calculate the null space using a QRD instead of an SVD. Thus, a natural way to reduce the complexity of the SVD-based method is to find a basis of $\mathcal{N}\left(\overline{\boldsymbol{H}}_{k}\right)$ using the QRD, instead of the SVD. The complexity of this method is further reduced in the first proposed design, where the recursive structure of $\overline{\boldsymbol{H}}_{k}$ is exploited. More specifically, a basis of $\mathcal{N}\left(\overline{\boldsymbol{H}}_{k}\right)$ can be computed iteratively from that of $\mathcal{N}\left(\overline{\boldsymbol{H}}_{k-1}\right)$. The second proposed method, which will be shown to require the lowest complexity, results from applying a QRD to a matrix composed by the channel matrices of all users. In this method, a single QRD is needed to find $\boldsymbol{B}_{k}$, instead of separately computing $\mathcal{N}\left(\overline{\boldsymbol{H}}_{k}\right)$ to find $\boldsymbol{B}_{k}$ for each $k$ as in the original and the first proposed designs. Noticeably, since columns of $\boldsymbol{B}_{k}$ in the second method do not span $\mathcal{N}\left(\overline{\boldsymbol{H}}_{k}\right)$, the optimality of this method for SZF-DPC should be investigated. In fact, we will prove that this proposed method is also an optimal precoder design for SZF-DPC.

The remainder of the paper is organized as follows. In Section II, we briefly review the SZF-DPC scheme and the SVDbased precoder design. In Section III, we present two optimal precoder designs, and analyze their computational complexity. 
Numerical results are given in Section IV, followed by some conclusions in Section V.

Notation: Bold lower and upper case letters represent vectors and matrices, respectively; $\boldsymbol{H}^{H}$ and $\boldsymbol{H}^{T}$ are Hermitian and normal transpose of $\boldsymbol{H}$, respectively; $\|\boldsymbol{H}\|_{F}$ and $|\boldsymbol{H}|$ are the Frobenius norm and determinant of $\boldsymbol{H}$, respectively. $\boldsymbol{I}_{M}$ represents an $M \times M$ identity matrix. $\mathcal{R}(\boldsymbol{H})$ and $\mathcal{N}(\boldsymbol{H})$ denote the column space and the null space of $\boldsymbol{H}$, respectively.

\section{SZF-DPC AND PRECODER DESIGN}

\section{A. $S Z F-D P C$}

Consider a single-cell MIMO BC with a base station (BS) and $K$ multiple-antenna users. The channel between the BS and the $k$ th user is modeled by a matrix $\boldsymbol{H}_{k} \in \mathbb{C}^{n_{k} \times N}$, where $N$ and $n_{k} \geqslant 1$ are the number of antennas at the BS and the $k$ th user, respectively. The received signal at the $k$ th user is given by

$$
\boldsymbol{y}_{k}=\boldsymbol{H}_{k} \boldsymbol{W}_{k} \boldsymbol{s}_{k}+\sum_{j<k} \boldsymbol{H}_{k} \boldsymbol{W}_{j} \boldsymbol{s}_{j}+\sum_{j>k} \boldsymbol{H}_{k} \boldsymbol{W}_{j} \boldsymbol{s}_{j}+\boldsymbol{n}_{k}
$$

where $\boldsymbol{W}_{k} \in \mathbb{C}^{N \times L_{k}}$ is the precoder, and $\boldsymbol{s}_{k} \in \mathbb{C}^{L_{k} \times 1}, L_{k} \leq$ $\min \left(N, n_{k}\right)$ is the vector of transmitted symbols of the $k$ th user, respectively. The background noise $\boldsymbol{n}_{k} \in \mathbb{C}^{n_{k} \times 1}$ is assumed to be white complex-Gaussian with zero mean and covariance matrix $\boldsymbol{I}_{n_{k}}$. It is well known that DPC is a capacity achieving transmission strategy for MIMO BCs. For the $k$ th user, the BS views the interference term $\sum_{j<k} \boldsymbol{H}_{k} \boldsymbol{W}_{j} \boldsymbol{s}_{j}$ as known non-causally, and can be perfectly eliminated by DPC. As a result, the resulting data rate of the $k$ th user is given by

$$
R_{k}^{\mathrm{DPC}}=\log \frac{\left|\boldsymbol{I}+\sum_{j \leq k}^{K} \boldsymbol{H}_{k} \boldsymbol{S}_{j} \boldsymbol{H}_{k}^{H}\right|}{\left|\boldsymbol{I}+\sum_{j<k} \boldsymbol{H}_{k} \boldsymbol{S}_{j} \boldsymbol{H}_{k}^{H}\right|},
$$

where $\boldsymbol{S}_{j}=\boldsymbol{W}_{j} \boldsymbol{W}_{j}^{H}$ is the transmit covariance matrix for the $j$ th user. Since (2) is non-convex with respect to $\boldsymbol{S}_{j}$, it is generally difficult to be handled. For the sum rate maximization problem for MIMO BCs under a total power constraint, several numerical algorithms have been proposed to find optimal $\left\{\boldsymbol{S}_{k}\right\}$ [2], [3]. However, these algorithms suffer from high computational complexity due to their iterative nature. In [4], SZF-DPC was proposed as a low-complexity alternative to DPC, which admits a closed-form solution for optimal precoders. In fact, SZF-DPC is a generalization of the zero-forcing DPC in [5], dedicated to single-antenna receivers. For SZF-DPC, the interference term $\sum_{j<k} \boldsymbol{H}_{k} \boldsymbol{W}_{j} \boldsymbol{s}_{j}$ in (1) is canceled by DPC, and the interference term $\sum_{j>k} \boldsymbol{H}_{k} \boldsymbol{W}_{j} \boldsymbol{s}_{j}$ is eliminated by designing $\boldsymbol{W}_{j}$ such that

$$
\boldsymbol{H}_{k} \boldsymbol{W}_{j}=\mathbf{0} \text { for all } j>k \text {. }
$$

Accordingly, the resulting data rate of the $k$ th user for SZFDPC is given by

$$
R_{k}^{\text {SZF-DPC }}=\log \left|\boldsymbol{I}+\boldsymbol{H}_{k} \boldsymbol{S}_{k} \boldsymbol{H}_{k}^{H}\right| .
$$

In this paper, we address the sum rate maximization problem for SZF-DPC under a sum power constraint $P$, which is formulated as

$$
\begin{array}{cl}
\underset{\boldsymbol{W}_{k}}{\operatorname{maximize}} & \sum_{k=1}^{K} \log \left|\mathbf{I}+\boldsymbol{H}_{k} \boldsymbol{W}_{k} \boldsymbol{W}_{k}^{H} \boldsymbol{H}_{k}^{H}\right| \\
\text { subject to } & \boldsymbol{H}_{j} \boldsymbol{W}_{k}=\mathbf{0}, \forall j<k \\
& \sum_{k=1}^{K} \operatorname{tr}\left(\boldsymbol{W}_{k} \boldsymbol{W}_{k}^{H}\right) \leq P .
\end{array}
$$

In [4], an approach to find the optimal precoders $\left\{\boldsymbol{W}_{k}\right\}$ for (5) was proposed using the SVD, which is described next.

\section{B. SVD-based design}

To solve (5), we can write $\boldsymbol{W}_{k}=\boldsymbol{B}_{k} \boldsymbol{D}_{k}$, where $\boldsymbol{B}_{k}$ is designed to remove the interference, and $\boldsymbol{D}_{k}$ is adjusted to maximize the sum rate under some power constraints. Obviously, the ZF constraints in (5) mean that $\boldsymbol{B}_{k}$ must lie in $\mathcal{N}\left(\overline{\boldsymbol{H}}_{k}\right)$, where $^{1}$

$$
\overline{\boldsymbol{H}}_{k}=\left[\begin{array}{llll}
\boldsymbol{H}_{1}^{H} & \boldsymbol{H}_{2}^{H} & \cdots & \boldsymbol{H}_{k-1}^{H}
\end{array}\right]^{H} \in \mathbb{C}^{\sum_{i=1}^{k-1} n_{i} \times N} .
$$

In [4], $\boldsymbol{B}_{k}$ is chosen to be an orthonormal basis of $\mathcal{N}\left(\overline{\boldsymbol{H}}_{k}\right)$, which can be found by an SVD of $\overline{\boldsymbol{H}}_{k}$. To be specific, denote the SVD of $\overline{\boldsymbol{H}}_{k}$ as

$$
\overline{\boldsymbol{H}}_{k}=\overline{\boldsymbol{U}}_{k} \overline{\boldsymbol{\Sigma}}_{k}\left[\overline{\boldsymbol{V}}_{k}^{(1)} \overline{\boldsymbol{V}}_{k}^{(0)}\right]^{H} .
$$

Then columns of $\overline{\mathbf{V}}_{k}^{(0)} \in \mathbb{C}^{N \times \bar{n}_{k}}, \bar{n}_{k}=N-\sum_{i=1}^{k-1} n_{i}$, form an orthonormal basis of $\mathcal{N}\left(\overline{\boldsymbol{H}}_{k}\right)$. To satisfy the ZF constraints, the SVD-based design simply chooses $\boldsymbol{B}_{k}=\overline{\boldsymbol{V}}_{k}^{(0)}$. The condition for the BS to support all users is that $\mathcal{N}\left(\overline{\boldsymbol{H}}_{k}\right)$ has a dimension larger than zero, for all $k$. Assuming the rows of all $\boldsymbol{H}_{k}$ 's are linearly independent, this requirement is equivalent to $N>\sum_{i=1}^{K-1} n_{i}$. When the number of users is large, a user scheduling algorithm is needed to choose a set of users that satisfies the above condition and can exploit the multiuser diversity gain [7]. In this paper, we assume $N>\sum_{i=1}^{K-1} n_{i}$ and focus on the precoder design. Since $\operatorname{tr}\left(\boldsymbol{W}_{k} \boldsymbol{W}_{k}^{H}\right)=\operatorname{tr}\left(\overline{\boldsymbol{V}}_{k}^{(0)} \boldsymbol{D}_{k} \boldsymbol{D}_{k}^{H}\left(\overline{\boldsymbol{V}}_{k}^{(0)}\right)^{H}\right)=\operatorname{tr}\left(\boldsymbol{D}_{k} \boldsymbol{D}_{k}^{H}\right)$, the sum rate maximization problem in (5) reduces to

$$
\begin{array}{cl}
\underset{\boldsymbol{D}_{k}}{\operatorname{maximize}} & \sum_{k=1}^{K} \log \left|\mathbf{I}+\tilde{\boldsymbol{H}}_{k} \boldsymbol{D}_{k} \boldsymbol{D}_{k}^{H} \tilde{\boldsymbol{H}}_{k}^{H}\right| \\
\text { subject to } & \sum_{k=1}^{K} \operatorname{tr}\left(\boldsymbol{D}_{k} \boldsymbol{D}_{k}^{H}\right) \leq P,
\end{array}
$$

where $\tilde{\boldsymbol{H}}_{k}=\boldsymbol{H}_{k} \overline{\boldsymbol{V}}_{k}^{(0)}$. Then, $\boldsymbol{D}_{k}$ can be easily found with water-filling over non-zero singular values of $\tilde{\boldsymbol{H}}_{k}$. More specifically, define a compact SVD of $\tilde{\mathbf{H}}_{k}$ as

$$
\tilde{\boldsymbol{H}}_{k}=\boldsymbol{H}_{k} \overline{\boldsymbol{V}}_{k}^{(0)}=\boldsymbol{U}_{k} \boldsymbol{\Sigma}_{k} \boldsymbol{V}_{k}^{H} \in \mathbb{C}^{n_{k} \times \bar{n}_{k}},
$$

where $\boldsymbol{\Sigma}_{k}$ is an $L_{k} \times L_{k}$ diagonal matrix that contains all non-zero singular values of $\tilde{\boldsymbol{H}}_{k}, L_{k}=\min \left(n_{k}, \bar{n}_{k}\right)$, and $\boldsymbol{V}_{k}$ represents the $L_{k}$ singular vectors of $\tilde{\boldsymbol{H}}_{k}$. To maximize the sum rate, $\boldsymbol{D}_{k}$ is found as $\boldsymbol{D}_{k}=\boldsymbol{V}_{k} \boldsymbol{\Phi}_{k}^{\frac{1}{2}}$, where $\boldsymbol{\Phi}_{k} \in \mathbb{C}^{L_{k} \times L_{k}}$ is a diagonal matrix, which is a solution to the following problem

$$
R^{\text {SZF-DPC }}=\underset{\boldsymbol{\Phi}_{k}: \sum \operatorname{tr}\left(\boldsymbol{\Phi}_{k}\right) \leq P}{\operatorname{maximize}} \sum_{k=1}^{K} \log _{2}\left|\mathbf{I}+\left(\boldsymbol{\Sigma}_{k}\right)^{2} \boldsymbol{\Phi}_{k}\right| .
$$

The set of optimal $\left\{\boldsymbol{\Phi}_{k}\right\}$ to (10) can be easily calculated using the water-filling algorithm. Finally, the precoder for the $k$ th

\footnotetext{
${ }^{1} \overline{\boldsymbol{H}}_{k}$ is only defined for $k \geq 2$ since we just need to find the precoding matrices $\boldsymbol{B}_{k}$ for $k \geq 2$. The precoding matrix $\boldsymbol{B}_{1}$ for the first user is set to be an identity matrix, i.e., $\boldsymbol{B}_{1}=\boldsymbol{I}_{N}$.
} 
user is given by $\boldsymbol{W}_{k}=\overline{\boldsymbol{V}}_{k}^{(0)} \boldsymbol{V}_{k} \boldsymbol{\Phi}_{k}^{\frac{1}{2}}$. Since the columns of $\overline{\boldsymbol{V}}_{k}^{(0)}$ span $\mathcal{N}\left(\overline{\boldsymbol{H}}_{k}\right)$, it is not difficult to see that the SVD-based method is optimal for (5). However, this method employs SVD to find the null space of a $\left(\sum_{i=1}^{k-1} n_{i}\right) \times N$ matrix for the $k$ th user, which is computationally costly.

\section{PROPOSED PRECODER DESIGNS}

In this section, we propose two optimal precoder design methods with significantly reduced complexity. We begin by introducing a simple method to reduce the complexity of the SVD-based design, where the SVD is replaced by the QRD, which has lower and deterministic complexity, to find an orthonormal basis of $\mathcal{N}\left(\overline{\boldsymbol{H}}_{k}\right)$. Specifically, applying a QRD to $\overline{\boldsymbol{H}}_{k}$ gives

$$
\overline{\boldsymbol{H}}_{k}=\left[\begin{array}{ll}
\boldsymbol{L}_{k} & \mathbf{0}
\end{array}\right]\left[\boldsymbol{Q}_{k}^{(1)} \boldsymbol{Q}_{k}^{(2)}\right]^{H},
$$

where $\boldsymbol{L} \in \mathbb{C}^{\sum_{i=1}^{k-1} n_{i} \times \sum_{i=1}^{k-1} n_{i}}$ is a lower triangular matrix, $\boldsymbol{Q}_{k}^{(1)} \in \mathbb{C}^{N \times \sum_{i=1}^{k-1} n_{i}}$ contains an orthonormal basis of $\mathcal{R}\left(\overline{\boldsymbol{H}}_{k}^{H}\right)$, and $\boldsymbol{Q}_{k}^{(2)} \in \mathbb{C}^{N \times \bar{n}_{k}}$ forms an orthonormal basis of $\mathcal{N}\left(\overline{\boldsymbol{H}}_{k}\right)$, i.e., $\overline{\boldsymbol{H}}_{k} \boldsymbol{Q}_{k}^{(2)}=\mathbf{0}$, and $\left(\boldsymbol{Q}_{k}^{(2)}\right)^{H} \boldsymbol{Q}_{k}^{(2)}=\boldsymbol{I}$. For brevity, we denote by null $(\boldsymbol{H})$ an orthonormal basis of $\mathcal{N}(\boldsymbol{H})$, e.g. $\operatorname{null}\left(\overline{\boldsymbol{H}}_{k}\right)=\boldsymbol{Q}_{k}^{(2)}$. In this simple method, we simply choose $\boldsymbol{B}_{k}=\boldsymbol{Q}_{k}^{(2)}$. Note that $\boldsymbol{Q}_{k}^{(2)}$ and $\overline{\boldsymbol{V}}_{k}^{(0)}$ are both orthonormal bases of $\mathcal{N}\left(\overline{\boldsymbol{H}}_{k}\right)$, and, thus, the two methods are equivalent. In the following, we present two precoder designs which have significantly lower complexity than the SVD-based method.

\section{A. Iterative QRD-based design (IQRD-based design)}

The above method can lower the complexity of the SVDbased method, but its complexity is still high because the QRD is applied to a matrix of large dimensions. From (6), we can write $\overline{\boldsymbol{H}}_{k+1}=\left[\begin{array}{ll}\overline{\boldsymbol{H}}_{k}^{H} & \boldsymbol{H}_{k}^{H}\end{array}\right]^{H}$. That is, $\overline{\boldsymbol{H}}_{k+1}$ and $\overline{\boldsymbol{H}}_{k}$ only differ by the channel matrix $\boldsymbol{H}_{k}$. Due to this structure of $\overline{\boldsymbol{H}}_{k+1}$, we can find $\mathcal{N}\left(\overline{\boldsymbol{H}}_{k+1}\right)$ efficiently based on the idea of intersection of null spaces [8]. Specifically, we have $\mathcal{N}\left(\overline{\boldsymbol{H}}_{k+1}\right)=\mathcal{N}\left(\overline{\boldsymbol{H}}_{k}\right) \cap \mathcal{N}\left(\boldsymbol{H}_{k}\right)$. Thus, columns of $\boldsymbol{B}_{k+1}$ should lie in the intersection of $\mathcal{N}\left(\overline{\boldsymbol{H}}_{k}\right)$ and $\mathcal{N}\left(\boldsymbol{H}_{k}\right)$. Assuming $\boldsymbol{B}_{k}=\operatorname{null}\left(\overline{\boldsymbol{H}}_{k}\right)$ is already known, we can design $\boldsymbol{B}_{k+1}$ as

$$
\boldsymbol{B}_{k+1}=\boldsymbol{B}_{k} \times \operatorname{null}\left(\boldsymbol{H}_{k} \boldsymbol{B}_{k}\right)
$$

In this way, $\boldsymbol{B}_{k+1}$ is recursively calculated from $\boldsymbol{B}_{k}$, the precoding matrix of the $k$ th user, and a QRD to $\boldsymbol{H}_{k} \boldsymbol{B}_{k}$, which has lower dimensions than $\overline{\boldsymbol{H}}_{k}$ in (6) since the size of $\boldsymbol{B}_{k}$ decreases after each step. Computing $\operatorname{null}\left(\boldsymbol{H}_{k} \boldsymbol{B}_{k}\right)$ is more computationally efficient than computing $\operatorname{null}\left(\overline{\boldsymbol{H}}_{k}\right)$. The IQRD-based method works as follows. The precoding matrix of the second user is computed as $\boldsymbol{B}_{2}=\operatorname{null}\left(\boldsymbol{H}_{1}\right) \in$ $\mathbb{C}^{N \times\left(N-n_{1}\right)}$. Next, the precoding matrix of user 3 is given by $\boldsymbol{B}_{3}=\boldsymbol{B}_{2} \times \operatorname{null}\left(\boldsymbol{H}_{2} \boldsymbol{B}_{2}\right) \in \mathbb{C}^{N \times\left(N-\sum_{i=1}^{2} n_{i}\right)}$. This recursive process is repeated until all precoding matrices are found. Note that $\boldsymbol{B}_{k}$ in the IQRD-based design also forms an orthonormal basis for $\mathcal{N}\left(\overline{\boldsymbol{H}}_{k}\right)$, and, thus, the IQRD-based design is an optimal alternative design for SZF-DPC.

\section{B. Generalized QRD-based design (GQRD-based design)}

The precoder designs mentioned above require to compute a basis of $\mathcal{N}\left(\overline{\boldsymbol{H}}_{k}\right)$ for each $k$. Due to the concatenated structure of $\overline{\boldsymbol{H}}_{k}$, the complexity of these methods increases with $k$. In this subsection, we propose a precoder design based on a single QRD. First, stack the channel matrix of all users in a matrix $\boldsymbol{H}$ defined as

$$
\boldsymbol{H}=\left[\begin{array}{llll}
\boldsymbol{H}_{1}^{H} & \boldsymbol{H}_{2}^{H} & \cdots & \boldsymbol{H}_{K}^{H}
\end{array}\right]^{H} \in \mathbb{C}^{n_{R} \times N},
$$

where $n_{R}=\sum_{k=1}^{K} n_{k}$ is the total number of receive antennas, and all precoders in a matrix $W$ given by

$$
\boldsymbol{W}=\left[\boldsymbol{W}_{1} \boldsymbol{W}_{2} \cdots \boldsymbol{W}_{K}\right] \in \mathbb{C}^{N \times L_{R}} .
$$

where $L_{R}=\sum_{k=1}^{K} L_{k}$ is the total number of data streams that the BS is able to transmit to all users in the system. Clearly, the product $\boldsymbol{H} \boldsymbol{W}$ has to be a lower block triangular matrix to satisfy the ZF constraints in (3). In the GQRD-based design, we further force $\boldsymbol{H} \boldsymbol{W}$ to be a lower triangular matrix. Specifically, consider a QRD of $\boldsymbol{H}$ given in (13) as

$$
\boldsymbol{H}=\boldsymbol{L} \boldsymbol{Q},
$$

and partition $L$ into

$$
\boldsymbol{L}=\left[\begin{array}{ccccc}
\boldsymbol{L}_{1} & & & & \\
\times & \boldsymbol{L}_{2} & & & \\
\times & \times & \ddots & & \\
\times & \times & \times & \ddots & \\
\times & \times & \times & \times & \boldsymbol{L}_{K}
\end{array}\right]
$$

where $\boldsymbol{L}_{k} \in \mathbb{C}^{n_{k} \times n_{k}}$ is a lower triangular matrix, and $\boldsymbol{Q}$ into

$$
\boldsymbol{Q}=\left[\begin{array}{llll}
\boldsymbol{Q}_{1} & \boldsymbol{Q}_{2} & \cdots & \boldsymbol{Q}_{K}
\end{array}\right]^{H}
$$

where $\boldsymbol{Q}_{k} \in \mathbb{C}^{N \times n_{k}}$ satisfies $\boldsymbol{H}_{j} \boldsymbol{Q}_{k}=\mathbf{0}, \forall j<k$, i.e., $\overline{\boldsymbol{H}}_{k} \boldsymbol{Q}_{k}=\mathbf{0}$, and $\boldsymbol{Q}_{k}^{H} \boldsymbol{Q}_{k}=\boldsymbol{I}_{n_{k}}$. To satisfy the ZF constraints, we design the precoding matrix $\boldsymbol{B}_{k}=\boldsymbol{Q}_{k}$. One possibility to compute the power allocation matrix $\boldsymbol{D}_{k}$ is to use the water-filling algorithm over $\left\{\operatorname{diag}\left(\boldsymbol{L}_{k}\right)\right\}$. This is equivalent to treating each receive antenna of each user as a separate user, and applying the QRD method in [5]. However, this approach is suboptimal since the rows of $\boldsymbol{H}_{k}$ should be jointly optimized. In the GQRD-based design, $\boldsymbol{D}_{k}$ is found, following (9) and (10) with $\tilde{\boldsymbol{H}}_{k}$ given by

$$
\tilde{\boldsymbol{H}}_{k}=\boldsymbol{H}_{k} \boldsymbol{Q}_{k}=\boldsymbol{L}_{k} \in \mathbb{C}^{n_{k} \times n_{k}} .
$$

That is, $\boldsymbol{D}_{k}$ is calculated from the singular values of $\boldsymbol{L}_{k}$, rather than from the diagonal elements of $\boldsymbol{L}_{k}$.

Comparing the effective channel matrices of the $k$ th user obtained from the SVD-based design in (9) and the GQRDbased design in (18), the former is an $n_{k} \times \bar{n}_{k}$ matrix, while the latter is an $n_{k} \times n_{k}$ square matrix. For $N>n_{R}$, it holds that $\bar{n}_{k}>n_{k}{ }^{2}$ Thus, one may ask if the data rate of each user is the same for both design methods. Note that $\boldsymbol{Q}_{k}$ is not a basis of $\mathcal{N}\left(\overline{\boldsymbol{H}}_{k}\right)$. More specifically, $\boldsymbol{Q}_{k}$ just lies in a subspace spanned by columns of $\boldsymbol{V}_{k}^{(0)}$. Consequently, the GQRD-based method

\footnotetext{
${ }^{2}$ Moreover, if $n_{R} \geq N>\sum_{i=1}^{K-1} n_{i}$, it also holds that $\bar{n}_{k} \geq n_{k}$ for $k=2, \ldots, K-1$. For the $K$ th user, i.e., the last user, $\boldsymbol{B}_{K}$ from both design methods have the same size of $n_{K} \times \bar{n}_{K}$
} 
seems to be a suboptimal solution for SZF-DPC. However, we now prove that the data rate of each user in the GQRD-based method is equal to that in the SVD-based method, and, thus, the GQRD-based method is also an optimal design for SZFDPC. Apparently, it is sufficient to show that the non-zero singular values of $\boldsymbol{H}_{k} \boldsymbol{V}_{k}^{(0)}$ and $\boldsymbol{H}_{k} \boldsymbol{Q}_{k}$ are the same. In fact, we prove a stronger result which is stated in the following theorem.

Theorem 1. The effective channel matrices for user $k$ obtained from the SVD-based design in (9) and the GQRD-based design in (18) satisfy $\boldsymbol{H}_{k} \boldsymbol{Q}_{k} \boldsymbol{Q}_{k}^{H} \boldsymbol{H}_{k}^{H}=$ $\boldsymbol{H}_{k} \overline{\boldsymbol{V}}_{k}^{(0)}\left(\overline{\mathbf{V}}_{k}^{(0)}\right)^{H} \boldsymbol{H}_{k}^{H}$.

Proof: Please refer to the Appendix.

Remark 1. As a special case when $n_{k}=1$ for all $k$, the GQRD-based method is identical to the beamformer design proposed in [5]. Consequently, Theorem 1 implies that the precoder design based on QRD in [5] is optimal for ZF-DPC in MISO BCs under a sum power constraint. This result is also established in [6] by another method.

\section{Complexity comparison}

In this subsection, we study the complexity of the precoder designs for SZF-DPC schemes presented above. Since the process of finding $\boldsymbol{D}_{k}$ is the same for all methods, we only consider the complexity of calculating $\boldsymbol{B}_{k}$. The complexity is measured by the number of flops as in [9], [10], denoted as $\psi .^{3}$ Although flop counting is a crude measurement of the true computational complexity, it captures the order of the computation load.

For simplicity, we analyze the complexity for the case where all users have the same number of antennas, i.e., $n_{k}=\bar{n}$, for all $k$. The number of supportable users is then given by $K=N / \bar{n}$, where $N$ is the number of transmit antennas at the BS, assumed to be a multiple of $\bar{n}$. The number of flops of some typical operations is given as follows. Multiplication of an $m \times p$ matrix and a $p \times n$ matrix requires $8 m p n$ flops. The number of flops needed to compute QRD of a real matrix of size $m \times n, m \geq n$ with fast Given transformations is $2 n^{2}(m-n / 3)$ [8]. For a complex matrix of the same size, we approximate the number of flops that involve in a QRD by $4 n^{2}(3 m-n)$, i.e., treating every operation as a complex multiplication. Similarly, the number of flops for an SVD of an $m \times n, m>n$, complex-valued matrix is approximated by $24 m n^{2}+48 m^{2} n+54 m^{3}[9]$.

1) SVD-based design: For the $k$ th user, $k \geq 2$, the number of flops needed to compute the SVD of $\overline{\boldsymbol{H}}_{k} \in \mathbb{C}^{(k-1) \bar{n} \times N}$ is $24(k-1) \bar{n} N^{2}+48(k-1)^{2} \bar{n}^{2} N+54(k-1)^{3} \bar{n}^{3}$. Thus, the

\footnotetext{
${ }^{3} \mathrm{~A}$ flop is equal to a real floating point operation [8]. A real addition, multiplication, or division is counted as one flop. A complex addition and multiplication have two flops and six flops, respectively.
}

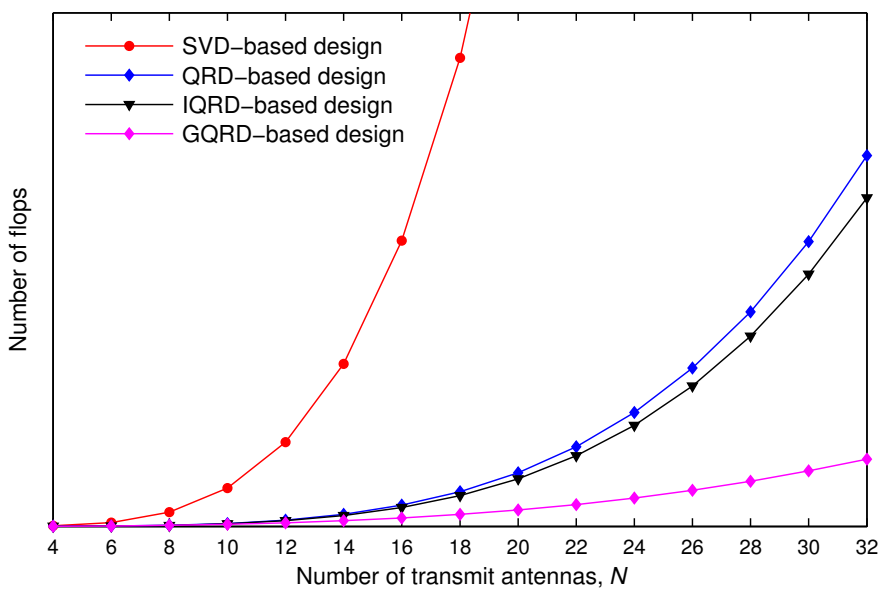

Fig. 1. Complexity comparison of various precoder designs for SZF-DPC schemes, $n_{k}=\bar{n}=2$.

total number of flops of the SVD-based precoder design is

$$
\begin{aligned}
\psi_{\mathrm{SVD}} \approx & \sum_{k=2}^{K}\left\{24(k-1) \bar{n} N^{2}+48(k-1)^{2} \bar{n}^{2} N\right. \\
& \left.+54(k-1)^{3} \bar{n}^{3}\right\} \\
\approx & 41 K N^{3} .
\end{aligned}
$$

2) IQRD-based design: To compute $\boldsymbol{B}_{2}=\operatorname{null}\left(\boldsymbol{H}_{1}\right)$, we need $4 \bar{n}^{2}(3 N-\bar{n})$ flops. For each $k \geq 3$, we need $8 \bar{n} N(N-(k-1) \bar{n})+4 \bar{n}^{2}(3(N-(k-1) \bar{n})-\bar{n})$ flops to obtain $\operatorname{null}\left(\boldsymbol{H}_{k-1} \boldsymbol{B}_{k-1}\right)$. It then takes $8 N(N-(k-2) \bar{n})(N-(k-$ 1) $\bar{n}$ ) flops to calculate the precoding matrix $\boldsymbol{B}_{k}$. Therefore, the resulting total number of flops for the IQRD-based design is

$$
\begin{aligned}
\psi_{\mathrm{IQRD}} \approx & 4 \bar{n}^{2}(3 N-\bar{n})+\sum_{k=3}^{K}\{8 \bar{n} N(N-(k-1) \bar{n}) \\
& +4 \bar{n}^{2}(3(N-(k-1) \bar{n})-\bar{n}) \\
& +8 N(N-(k-2) \bar{n})(N-(k-1) \bar{n})\} \\
\approx & 9 / 4 K N^{3} .
\end{aligned}
$$

3) GQRD-based design: The complexity of the generalized QRD-based design is equivalent to the number of flops needed to compute a QRD of $\boldsymbol{H}$, which is given by

$$
\psi_{\mathrm{GQRD}}=4 N^{2}(3 N-N)=8 N^{3} \text {. }
$$

\section{NUMERICAL RESULTS}

In this section, we provide numerical examples to demonstrate the results in this paper. First, the complexity of the precoder designs presented above is compared in Fig. 1, where we plot the number of flops versus the number of transmit antennas, $N$. In Fig. 1, the number of receive antennas is the same for all users, $n_{k}=\bar{n}=2$, for all $k$, and the number of users is $K=N / \bar{n}$. The label 'QRD-based design' refers to the simple method, in which the SVD is replaced by the QRD. As we can see, the IQRD-based design requires slightly lower complexity than the QRD-based method. Noticeably, the GQRD-based precoder design greatly reduces the complexity of other precoder designs.

To numerically verify the optimality of the two proposed 


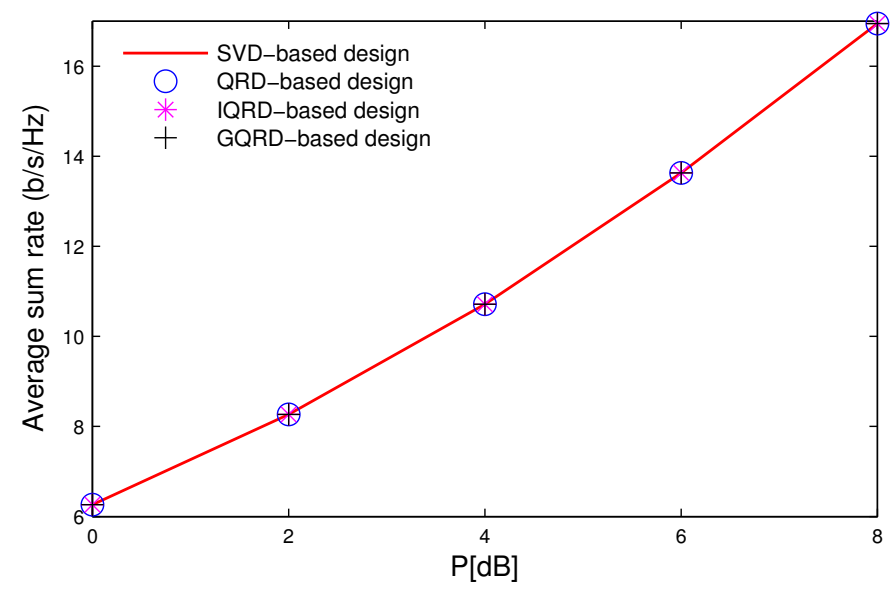

Fig. 2. Sum rate comparison of precoder designs for SZF-DPC, $N=8, K=$ $4, n_{k}=2, \forall k=1,2, \ldots, 4$.

precoder designs, we plot the average sum rate of the SVDbased design and the two proposed precoder designs as a function of $P$, the total transmit power in Fig. 2. A quasi-static fading model is used in our simulation, where independent realizations of $\boldsymbol{H}_{k}$ are generated as zero mean and unit variance complex Gaussian random variables for each snapshot. Fig. 2 considers a downlink channel with $N=8, K=4$, and $n_{i}=2$ for $i=1,2, \ldots, 4$. The simulation results in Fig. 2 again confirm that all precoder designs proposed in this paper have the same sum rate, which is equal to that of the SVDbased method.

\section{Conclusions}

This paper addresses the precoder design for SZF-DPC. In particular, we propose two low-complexity optimal precoder designs under a sum power constraint. The proposed precoder designs are shown to be optimal and have different degrees of computational complexity, which is lower than the existing method using the SVD.

\section{APPENDIX}

\section{PROOF OF THEOREM 1}

In this appendix, we show that $\boldsymbol{H}_{k} \boldsymbol{Q}_{k} \boldsymbol{Q}_{k}^{H} \boldsymbol{H}_{k}^{H}=$ $\boldsymbol{H}_{k} \overline{\boldsymbol{V}}_{k}^{(0)}\left(\overline{\mathbf{V}}_{k}^{(0)}\right)^{H} \boldsymbol{H}_{k}^{H}$. By definition, we have $\overline{\boldsymbol{V}}_{1}^{(0)}=\boldsymbol{I}_{N}$. From the QRD of $\boldsymbol{H}$, it is easy to check that $\boldsymbol{H}_{1}=$ $\boldsymbol{L}_{1} \boldsymbol{Q}_{1}^{H}$, and that $\boldsymbol{H}_{1} \boldsymbol{B}_{1} \boldsymbol{B}_{1}^{H} \boldsymbol{H}_{1}^{H}=\boldsymbol{L}_{1} \boldsymbol{L}_{1}^{H}=\boldsymbol{H}_{1} \boldsymbol{H}_{1}^{H}=$ $\boldsymbol{H}_{1} \overline{\boldsymbol{V}}_{1}^{(0)}\left(\overline{\boldsymbol{V}}_{1}^{(0)}\right)^{H} \boldsymbol{H}_{1}^{H}$. Thus, it remains to prove Theorem 1 for $k \geq 2$. First, according to the SVD-based method, the columns of $\overline{\boldsymbol{V}}_{k}^{(0)}$ form an orthogonal basis of $\mathcal{N}\left(\overline{\boldsymbol{H}}_{k}\right)$. Since $\overline{\boldsymbol{H}}_{k} \boldsymbol{Q}_{k}=\mathbf{0}$, i.e., $\boldsymbol{Q}_{k}$ lies in $\mathcal{N}\left(\overline{\boldsymbol{H}}_{k}\right), \boldsymbol{Q}_{k}$ can be obtained from $\overline{\boldsymbol{V}}_{k}^{(0)}$ through a linear transformation. That is, there exists a matrix $\boldsymbol{\Psi} \in \mathbb{C}^{\bar{n}_{k} \times n_{k}}$ such that $\boldsymbol{\Psi}^{H} \boldsymbol{\Psi}=\boldsymbol{I}_{n_{k}}$ and

$$
\overline{\boldsymbol{V}}_{k}^{(0)} \boldsymbol{\Psi}=\boldsymbol{Q}_{k}
$$

From the GQRD-based design, we also have

$$
\boldsymbol{H}_{k} \boldsymbol{Q}_{k}=\boldsymbol{L}_{k}
$$

where $\boldsymbol{L}_{k} \in \mathbb{C}^{n_{k} \times n_{k}}$ is a lower triangular matrix. Next, consider the LQD of $\boldsymbol{H}_{k} \overline{\boldsymbol{V}}_{k}^{(0)}$ which is given by

$$
\boldsymbol{H}_{k} \overline{\boldsymbol{V}}_{k}^{(0)}=\overline{\boldsymbol{L}}_{k} \overline{\boldsymbol{Q}}_{K}
$$

where $\overline{\boldsymbol{L}}_{k} \in \mathbb{C}^{n_{k} \times n_{k}}$ is lower triangular matrix, and $\overline{\boldsymbol{Q}}_{K} \in$ $\mathbb{C}^{n_{k} \times \bar{n}_{k}}$ has orthogonal rows, i.e., $\overline{\boldsymbol{Q}}_{K} \overline{\boldsymbol{Q}}_{K}^{H}=\boldsymbol{I}_{n_{k}}$. The LQD in (24) is the Gram-Schmidt orthogonalization of rows of $\boldsymbol{H}_{k} \overline{\boldsymbol{V}}_{k}^{(0)}$, started from the first row. Multiplying both sides of (24) by $\boldsymbol{\Psi}$ and using (22), we can write

$$
\boldsymbol{H}_{k} \boldsymbol{Q}_{k}=\boldsymbol{H}_{k} \overline{\boldsymbol{V}}_{k}^{(0)} \boldsymbol{\Psi}=\overline{\boldsymbol{L}}_{k} \overline{\boldsymbol{Q}}_{K} \boldsymbol{\Psi}
$$

To prove Theorem 1, we now proceed to show that $\boldsymbol{L}_{k}=\overline{\boldsymbol{L}}_{k}$ and $\overline{\boldsymbol{Q}}_{K} \boldsymbol{\Psi} \in \mathbb{C}^{n_{k} \times n_{k}}$ is a unitary matrix, i.e., $\boldsymbol{\Psi}^{H} \overline{\boldsymbol{Q}}_{K}^{H} \overline{\boldsymbol{Q}}_{K} \boldsymbol{\Psi}=$ I. As a result of the LQD of $\boldsymbol{H}_{k} \overline{\boldsymbol{V}}_{k}^{(0)}$ in (24), columns of $\overline{\boldsymbol{Q}}_{K}^{H}$ form an orthogonal basis for $\mathcal{R}\left(\left(\overline{\boldsymbol{V}}_{k}^{(0)}\right)^{H} \boldsymbol{H}_{k}^{H}\right)$, and, thus, $\overline{\boldsymbol{Q}}_{K}^{H} \overline{\boldsymbol{Q}}_{K}$ is the orthogonal projector onto $\mathcal{R}\left(\left(\overline{\boldsymbol{V}}_{k}^{(0)}\right)^{H} \boldsymbol{H}_{k}^{H}\right)$. From (22), we have $\boldsymbol{\Psi}=$ $\left(\overline{\boldsymbol{V}}_{k}^{(0)}\right)^{H} \boldsymbol{Q}_{k}$, and $\boldsymbol{\Psi} \boldsymbol{\Psi}^{H}$ is the orthogonal projector onto $\mathcal{R}\left(\left(\overline{\boldsymbol{V}}_{k}^{(0)}\right)^{H} \boldsymbol{Q}_{k}\right)$. Since (23) holds, it is easy to see that $\mathcal{R}\left(\left(\overline{\boldsymbol{V}}_{k}^{(0)}\right)^{H} \boldsymbol{H}_{k}^{H}\right)=\mathcal{R}\left(\left(\overline{\boldsymbol{V}}_{k}^{(0)}\right)^{H} \boldsymbol{Q}_{k}\right)$, and, thus, $\overline{\boldsymbol{Q}}_{K}^{H} \overline{\boldsymbol{Q}}_{K}=$ $\boldsymbol{\Psi} \boldsymbol{\Psi}^{H}$ due to the uniqueness of orthogonal projector onto the same space. Accordingly, $\boldsymbol{\Psi}^{H} \overline{\boldsymbol{Q}}_{K}^{H} \overline{\boldsymbol{Q}}_{K} \boldsymbol{\Psi}=\boldsymbol{\Psi}^{H} \boldsymbol{\Psi} \boldsymbol{\Psi}^{H} \boldsymbol{\Psi}=\mathbf{I}$. We can observe that both (23) and (25) are in fact LQ decompositions of the same square matrix $\boldsymbol{H}_{k} \boldsymbol{Q}_{k}$. It follows by the uniqueness of the LQ decomposition of $\boldsymbol{H}_{k} \boldsymbol{Q}_{k}$ that

$$
\begin{aligned}
\overline{\boldsymbol{L}}_{k} & =\boldsymbol{L}_{k} \\
\overline{\boldsymbol{Q}}_{K} \boldsymbol{\Psi} & =\boldsymbol{I}_{n_{k}}
\end{aligned}
$$

Combining (23), (24), and (26) gives $\boldsymbol{H}_{k} \overline{\boldsymbol{V}}_{k}^{(0)}\left(\overline{\boldsymbol{V}}_{k}^{(0)}\right)^{H} \boldsymbol{H}_{k}^{H}=$ $\overline{\boldsymbol{L}}_{k} \overline{\boldsymbol{L}}_{k}^{H}=\boldsymbol{L}_{k} \boldsymbol{L}_{k}^{H}=\boldsymbol{H}_{k} \boldsymbol{Q}_{k} \boldsymbol{Q}_{k}^{H} \boldsymbol{H}_{k}^{H}$, and, thus, completes the proof.

\section{REFERENCES}

[1] H. Weingarten, Y. Steinberg, and S. Shamai, "The capacity region of the Gaussian multiple-input multiple-output broadcast channel,' IEEE Trans. Inf. Theory, vol. 52, no. 9, pp. 3936-3964, Sep. 2006.

[2] N. Jindal, W. Rhee, S. Vishwanath, S. Jafar, and A. Goldsmith, "Sum power iterative water-filling for multi-antenna Gaussian broadcast channels," IEEE Trans. Inf. Theory, vol. 51, no. 4, pp. 1570-1580, Apr. 2005.

[3] W. Yu, "Sum-capacity computation for the Gaussian vector broadcast channel via dual decomposition," IEEE Trans. Inf. Theory, vol. 52, no. 2, pp. $754-759$, Feb. 2006.

[4] A. Dabbagh and D. Love, "Precoding for multiple antenna Gaussian broadcast channels with successive zero-forcing," IEEE Trans. Signal Process., vol. 55, no. 7, pp. 3837-3850, Jul. 2007.

[5] G. Caire and S. Shamai, "On the achievable throughput of a multiantenna Gaussian broadcast channel," IEEE Trans. Inf. Theory, vol. 49, no. 7, pp. 1691-1706, Jul. 2003.

[6] Le-Nam Tran, M. Juntti, M. Bengtsson, and B. Ottersten, "On the optimality of beamformer design for zero-forcing DPC with QR decomposition," IEEE ICC 2012, to appear.

[7] Le-Nam Tran and Een-Kee Hong, "Multiuser diversity for successive zero-forcing dirty paper coding: Greedy scheduling algorithms and asymptotic performance analysis," IEEE Trans. Signal Process., vol. 58, no. 6, pp. 3411-3416, Jun. 2010.

[8] G. H. Golub and C. F. V. Loan, Matrix Computations, 3rd ed. The John Hopkins Univ. Press, 1996.

[9] Z. Shen, R. Chen, J. Andrews, J. Heath, R.W., and B. Evans, "Low complexity user selection algorithms for multiuser MIMO systems with block diagonalization," IEEE Trans. Signal Process., vol. 54, no. 9, pp. 3658-3663, Sep. 2006.

[10] X. Zhang and J. Lee, "Low complexity MIMO scheduling with channel decomposition using capacity upperbound," IEEE Commun. Lett., vol. 56, no. 6, pp. 871-876, Jun. 2008. 